\title{
Why whole grains are protective: biological mechanisms
}

\author{
Joanne Slavin \\ Department of Food Science and Nutrition, University of Minnesota, 1334 Eckles Avenue, St Paul, MN 55108, USA
}

\begin{abstract}
Epidemiological studies find that whole-grain intake is protective against cancer, cardiovascular disease, diabetes and obesity. Potential mechanisms for this protection are diverse since whole grains are rich in nutrients and phytochemicals. First, whole grains are concentrated sources of dietary fibre, resistant starch and oligosaccharides, carbohydrates that escape digestion in the small intestine and are fermented in the gut, producing short-chain fatty acids (SCFA). SCFA lower colonic $\mathrm{pH}$, serve as an energy source for the colonocytes and may alter blood lipids. These improvements in the gut environment may provide immune protection beyond the gut. Second, whole grains are rich in antioxidants, including trace minerals and phenolic compounds, and these compounds have been linked to disease prevention. Additionally, whole grains mediate insulin and glucose responses. Although lower glycaemic load and glycaemic index have been linked to diabetes and obesity, risk of cancers such as colon and breast cancer have also been linked to high intake of readily-available carbohydrate. Finally, whole grains contain many other compounds that may protect against chronic disease. These compunds include phytate, phyto-oestrogens such as lignan, plant stanols and sterols, and vitamins and minerals. As a consequence of the traditional models of conducting nutrition studies on isolated nutrients, few studies exist on the biological effects of increased whole-grain intake. The few whole-grain feeding studies that are available show improvements in biomarkers with whole-grain consumption, such as weight loss, blood lipid improvement and antioxidant protection.
\end{abstract}

Whole grains: Bioactive compounds: Large bowel: Glucose and insulin: Antioxidants

Whole grains are rich in a wide range of compounds with known health effects (Fig. 1). When evaluating the mechanisms by which whole grains are protective against chronic disease, use can be made of the existing data describing mechanisms by which each of these individual nutrients and phytochemicals is protective. Additionally, it is clear that whole-grain consumption is protective beyond what would be predicted if the protection found with these individual compounds was just added up (Slavin et al. 2001a). Thus, there appears to be a synergy among the wide range of protective compounds in whole grains, suggesting that the whole is greater than the sum of the parts.

In the present paper four mechanisms that have been studied for the protectiveness of whole grains will be reviewed:

(a) large-bowel effects of whole grains;

(b) glucose and insulin changes seen with whole-grain consumption;

(c) the antioxidant theory for whole-grain protectiveness;

(d) other bioactive compounds in whole grains.

\section{Mechanistic studies of whole grains}

Despite dietary recommendations to increase intake of whole grains, little research has been conducted on the physiological effects of a diet high in whole grains. Generally, mechanistic studies have been conducted using the magic bullet approach, where one dietary ingredient is isolated and fed to either animals or human subjects. Thus, more published research supports a protective role for dietary fibre, trace minerals, vitamins or other nutrients, or phytochemicals than for the whole grain. Yet, epidemiological studies provide evidence that whole foods, including whole grains, are protective against a wide range of diseases, and generally this protectiveness is greater than that seen with any individual ingredient (Pereira et al. 2001).

Components in whole grains associated with improved health status include lignans, tocotrienols and phenolic compounds, and anti-nutrients such as phytic acid, tannins and enzyme inhibitors. In the grain-refining process the bran is removed, resulting in loss of dietary fibre, vitamins, minerals, lignans, phyto-oestrogens, phenolic compounds

Abbreviation: GI, glycaemic index.

Corresponding author: Dr Joanne Slavin, fax +1 612625 5272, email jslavin@umn.edu 


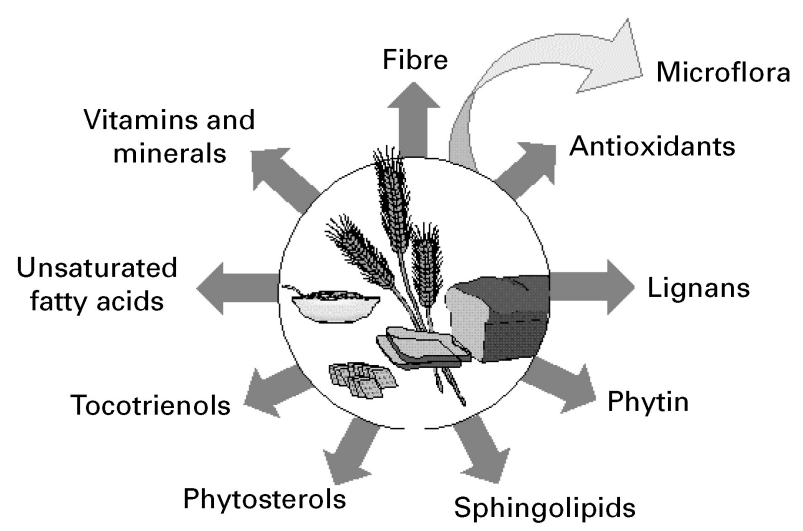

Fig. 1. Phytonutrient content of whole grains.

and phytic acid. Thus, in refined grains the relative concentration of starch is higher because most of the bran and some of the germ is removed in the refining process. The structure of all grains is similar and includes the endosperm, germ and bran. The absolute amount of each of these components varies among grains. For example, the bran content of maize is $6 \%$ while that of wheat is $16 \%$. Important grains in the US diet include wheat, rice, maize and oats.

To conduct feeding studies in human subjects whole grains must be fed in a form acceptable to the participants. Often, feeding studies use processed whole grains for the dietary intervention. Most research indicates that processing of whole grains does not remove biologically-important compounds (Slavin et al. 2001b). Additionally, epidemiological studies that report protection with whole-grain consumption relate whole-grain intake to processed wholegrain products, such as breads, cereals and brown rice.

\section{Large bowel effects of whole grains}

Whole grains are rich sources of fermentable carbohydrates, including dietary fibre, resistant starch and oligosaccharides. Undigested carbohydrate reaching the colon is fermented by intestinal microflora to short-chain fatty acids and gases. Short-chain fatty acids include acetate, butyrate and propionate, with butyrate being a preferred fuel for the colonic mucosa cells. Short-chain fatty acid production has been related to lowered serum cholesterol and decreased risk of cancer. Undigested carbohydrates increase faecal wet and dry weight and increase intestinal transit.

No studies have examined the effects of whole grains on gut fermentation. Research has been conducted on grain components, including dietary fibre, resistant starch and oligosaccharides. A comparison of the dietary fibre contents of various whole grains shows that oats, rye and barley contain about one-third soluble fibre and the rest is insoluble fibre. Soluble fibre is associated with cholesterol lowering and improved glucose response, while insoluble fibre is associated with improved bowel emptying. Wheat is lower in soluble fibre than most grains, while rice contains virtually no soluble fibre. Refining of grains results in a low total dietary fibre content and removes proportionally more of the insoluble fibre than soluble fibre.

Disruption of cell walls can increase the fermentability of dietary fibre. Coarse wheat bran has a greater faecal bulking effect than finely-ground wheat bran when fed at the same dosage (Wrick et al. 1983), suggesting that the particle size of the whole grain is an important factor in determining physiological effect. Coarse bran delays gastric emptying and accelerates small bowel transit. The effect seen with coarse bran was similar to that of inert plastic particles, suggesting that the coarse nature of whole grains as compared with refined grains has a unique physiological effect beyond composition differences between whole and refined grains (McIntyre et al. 1997).

Not all starch is digested and absorbed during gut transit. Factors that determine whether starch is resistant to digestion include the physical form of grains or seeds in which starch is located (particularly if they are whole or partially disrupted), size and type of starch granules, associations between starch and other dietary components, and cooking and food processing, especially cooking and cooling (Bjorck et al. 1994).

In addition to dietary fibre and resistant starch, grains contain substantial amounts of oligosaccharides. Oligosaccharides are defined as carbohydrates with a low (2-20) extent of polymerization. Common oligosaccharides include oligofructose and inulin. Wheat flour contains $10-40 \mathrm{mg}$ fructans/g dry weight (Van Loo et al. 1995). Fructans have also been found in rye and barley, with very young barley kernels containing $220 \mathrm{mg}$ fructan/g. Van Loo et al. (1995) have estimated that wheat provides $78 \%$ of the North American intake of oligosaccharides. Oligosaccharides have similar effects to those of soluble dietary fibre in the human gut. Moreover, it has consistently been shown that oligosaccharides can alter the human faecal flora. Human studies (Gibson et al. 1995) indicate that consumption of fructooligosaccharides increases bifidobacteria in the gut while decreasing concentrations of Escherichia coli, clostridia and bacteroides.

Although little research has been conducted directly on whole grains and bowel function, it is well known that dietary fibre from grains such as wheat and oats increases stool weight and speed transit (Marlett et al. 2002). Furthermore, the ability of components in whole grains to alter the microflora has important implications in health and disease (Fig. 2).

\section{Glucose and insulin changes seen with whole-grain consumption}

It is well accepted that glucose and insulin are linked to chronic diseases, especially diabetes. Whole-grain consumption is part of a healthy diet described as the 'prudent' diet. Epidemiological studies consistently show that risk for type 2 diabetes mellitus is decreased with consumption of whole grains (Van Dam et al. 2002). Additionally, Giovannucci (1995) proposed that insulin and colon cancer are linked. He suggests that insulin is an important growth factor of colonic epithelial cells and is a mitogen of tumour cell growth in vitro. Epidemiological studies indicate a similarity of factors that produce elevated insulin levels with those related to colon cancer risk, including obesity and low physical activity. Schoen et al. (1999) found that incident colon cancer was linked to higher levels of blood glucose and insulin and larger body weight. Hu et al. (1999) noted 


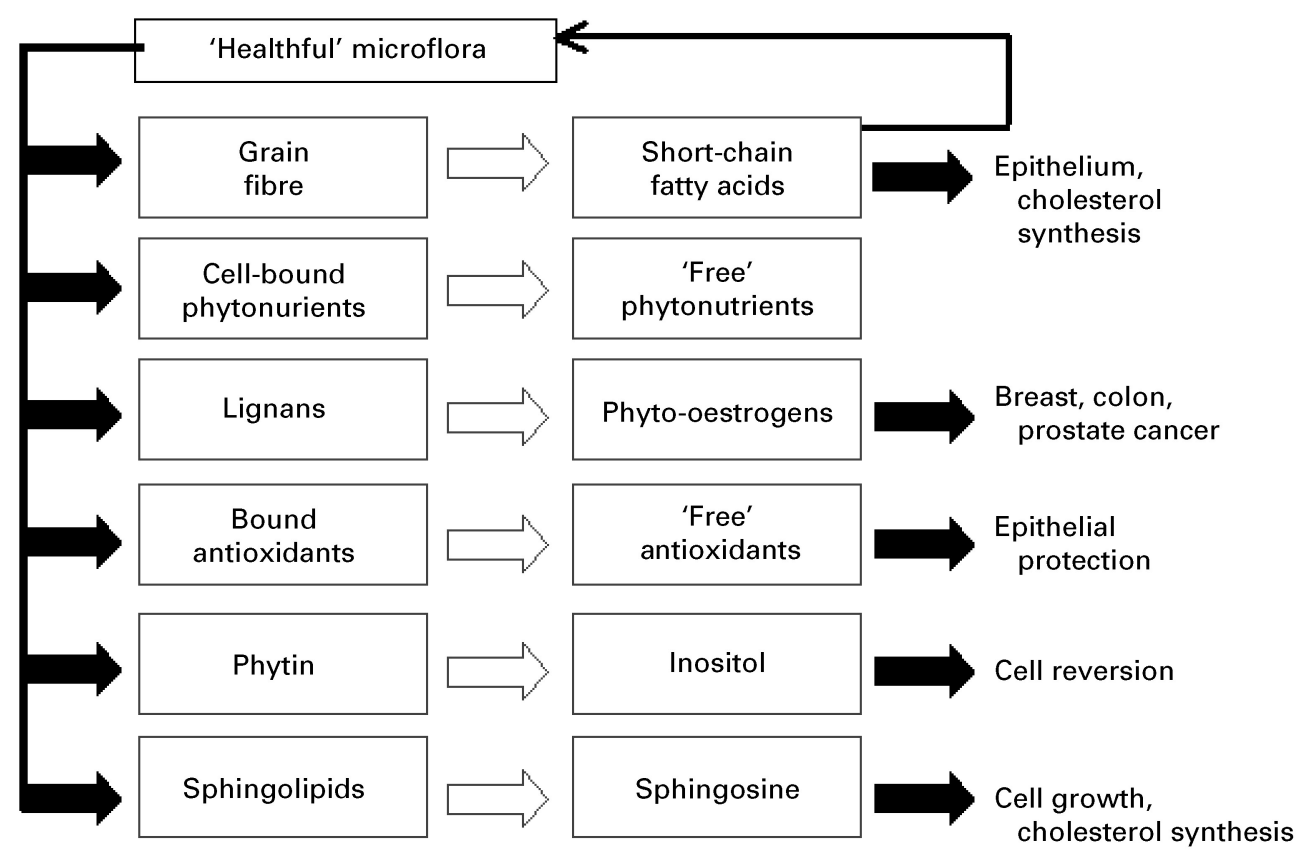

Fig. 2. Interactions between components in whole grains and the microflora that have important implications in health and disease.

the similarity of lifestyle and environmental risk factors for type 2 diabetes and colon cancer. They examined the relationship between diabetes and risk of colo-rectal cancer in the Nurses' Health Study, and found that they were related, with women with diabetes having increased risk of colo-rectal cancer.

To determine the relationship between whole-grain intake and glucose and insulin metabolism requires biomarkers. Glycaemic index (GI) is one marker used to compare the glycaemic response to foods. The GI is defined as the incremental area under the blood glucose response curve for the test food divided by the corresponding area after an equicarbohydrate portion of white bread, multiplied by 100 . It is known that glycaemic response is affected by many physiological factors. Other factors affecting the response include the form of the food, cooking, processing, fat content of the food and soluble fibre content of the food.

Whole foods are also known to slow digestion and absorption of carbohydrates. Postprandial blood glucose and insulin responses are greatly affected by food structure. Any process that disrupts the physical or botanical structure of food ingredients will increase the plasma glucose and insulin responses. Food structure has been found to be more important than gelatinization or the presence of viscous dietary fibre in determining glycaemic response (Granfeldt et al. 1995). Another study suggested the importance of preserved structure in foods as an important determinant of glycaemic response in diabetic subjects (Jarvi et al. 1995). Refining grains tends to increase glycaemic response and, thus, whole grains should slow glycaemic response (Jenkins et al. 1986).

Intact whole grains of barley, rice, rye, oats, maize, buckwheat and wheat have GI of 36-81, with barley and oats having the lowest values (Jenkins et al. 1988). Lower blood glucose levels and decreased insulin secretion have been observed in both normal and diabetic subjects while consuming a low-GI (67) diet containing pumpernickel bread with intact whole grains, bulgar (parboiled wheat), pasta and legumes compared with a high-GI (90) diet containing white bread and potato.

Heaton et al. (1988) compared glucose response when subjects consumed whole grains, cracked grains, wholegrain flour, and refined grain flour. Plasma insulin responses increased stepwise, with whole grains $<$ cracked grains $<$ coarse flour $<$ fine flour. Oat-based meals evoked smaller glucose and insulin responses than wheat- or maize-based meals. Particle size influenced the digestion rate and consequent metabolic effects of wheat and maize, but not oats. The authors suggested that the increased insulin response to finely-ground flour may be relevant to the aetiology of diseases associated with hyperinsulinaemia and to the management of diabetes.

Some feeding studies have been conducted to evaluate the relationship between whole grains and glucose metabolism. Pereira et al. (2002) tested the hypothesis that whole-grain consumption improves insulin sensitivity in overweight and obese adults. Eleven overweight or obese hyperinsulinaemic adults aged 25-56 years consumed two diets, each for 6 weeks. Diets were identical, except that refined-grain products were replaced with wholegrain products. At the end of each treatment, subjects consumed $355 \mathrm{ml}$ of a liquid mixed meal and blood samples were taken over $2 \mathrm{~h}$. Fasting insulin was $10 \%$ lower during consumption of the wholegrain diet. The authors concluded that insulin sensitivity might be an important mechanism whereby wholegrain foods reduce the risk of type 2 diabetes and heart disease.

Juntunen et al. (2002) evaluated the factors in grain products that affect human glucose and insulin responses. They fed the following grain products: whole-kernel rye bread; wholemeal rye bread containing oat $\beta$-glucan concentrate; dark durum wheat pasta; wheat bread made 
from white wheat flour. Glucose responses and the rate of gastric emptying after consumption of the two rye breads and pasta did not differ from those after consumption of white wheat bread. Insulin, glucose-dependent insulinotropic polypeptide and glucagon-like peptide 1 levels were lower after consumption of rye breads and pasta than after consumption of white wheat bread. These results confirm that postprandial insulin responses to grain products are determined by the form of food and by botanical structure not by the amount of fibre or the type of cereal in the food.

Other feeding studies have looked at different biomarkers relevant to cardiovascular disease. Truswell (2002) concluded that there is enough evidence to indicate that wholegrain products may reduce the risk of CHD. Katz et al. (2001) measured the effect of oat and wheat cereals on endothelial responses in human subjects. They report that daily supplementation with either wholegrain oat or wheat cereal for 1 month may prevent postprandial impairment of vascular reactivity in response to a high-fat meal. In a randomized controlled clinical trial consumption of wholegrain and legume powder reduced insulin demand, lipid peroxidation and plasma homocysteine concentrations in patients with coronary artery disease (Jang et al. 2001). Finally, consumption of wholegrain oat cereal was associated with improved blood pressure control and reduced the need for anti-hypertensive medications (Pins et al. 2002). Thus, clinical studies to date suggest that wholegrain consumption can improve biomarkers relevant to diabetes and cardiovascular disease.

\section{Antioxidant theory for whole-grain protectiveness}

The primary protective function of antioxidants in the body is their reaction with free radicals. Free radical attack on DNA, lipids and protein is thought to be an initiating factor for several chronic diseases (Miller, 2001). Cellular membrane damage from free radical attack and peroxidation is thought to be a primary causative factor. Cellular damage causes a shift in net charge of the cell, changing osmotic pressure, leading to swelling and eventual cell death. Free radicals also contribute to general inflammatory response and tissue damage. Antioxidants also protect DNA from oxidative damage and mutation that can lead to cancer. Free radical compounds result from normal metabolic activity as well as from the diet and environment. The body has defence mechanisms to prevent free radical damage and to repair damage, but when the defence is not sufficient, disease may develop. It follows that if dietary antioxidants reduce free radical activity in the body, then disease potential is reduced.

Wholegrain products are relatively high in antioxidant activity (Fig. 3). Antioxidants found in wholegrain foods are water-soluble and fat-soluble, and approximately half are insoluble. Soluble antioxidants include phenolic acids, flavonoids, tocopherols and avenanthramides in oats. A majority of the insoluble antioxidants are bound as cinnamic acid esters to arabinoxylan side chains of hemicellulose. Wheat bran insoluble fibre contains approximately $0 \cdot 5-1.0 \%$ phenolic groups. Covalently-bound phenolic acids are good free radical scavengers. About two-thirds of whole-grain antioxidant activity is not soluble in water, aqueous methanol

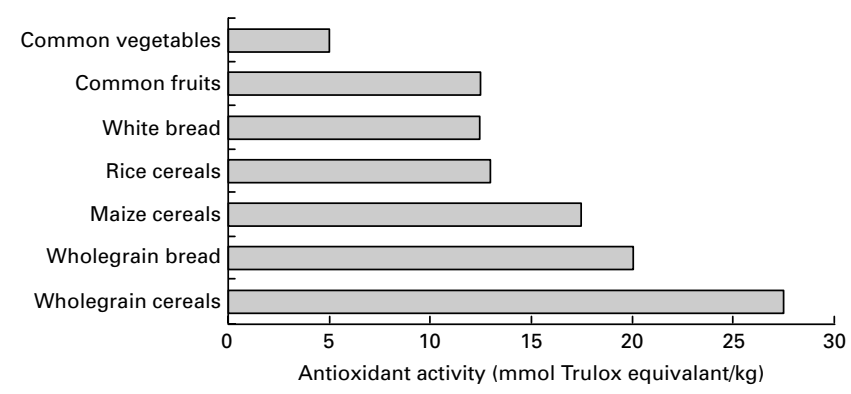

Fig. 3. Average antioxidant activity of vegetables, fruits and cereals determined using a diphenyl picrylhydrazyl radical-scavenging procedure.

or hexane. Antioxidant activity is an inherent property of insoluble grain fibre. In the colon hydrolysis by microbial enzymes frees bound phenolic acids (Kroon et al. 1997). Colon endothelial cells may absorb the released phenolic acids and gain antioxidant protection, and also these phenolic acids may enter the portal circulation. In this manner, wholegrain foods provide antioxidant protection over a long time period through the entire digestive tract.

In addition to natural antioxidants, antioxidant activity is created in grain-based foods by browning reactions during baking and toasting processes that increase total activity in the final product as compared with raw ingredients. For example, the crust of white bread has double the antioxidant activity of the starting flour or crust-free bread. Reductone intermediates from Maillard reactions may explain the increase in antioxidant activity. The total antioxidant activity of wholegrain products is similar to that of fruits or vegetables on a per serving basis (Miller, 2001).

Phytic acid, concentrated in grains, is a known antioxidant. Phytic acid forms chelates with various metals, which suppress damaging Fe-catalysed redox reactions. Colonic bacteria produce oxygen radicals in appreciable amounts and dietary phytic acid could suppress oxidant damage to the intestinal epithelium and neighbouring cells.

Vitamin $\mathrm{E}$ is another antioxidant present in whole grains that is removed in the refining process. Vitamin $E$ is an intracellular antioxidant that protects polyunsaturated fatty acids in cell membranes from oxidative damage. Another possible mechanism for vitamin E relates to its capacity to maintain $\mathrm{Se}$ in the reduced state. Vitamin $\mathrm{E}$ inhibits the formation of nitrosamines, especially at low $\mathrm{pH}$.

$\mathrm{Se}$ is another component that is removed in the refining process. Its content in food grains is proportional to the Se content of the soil in which the grain is grown. Se functions as a cofactor for glutathione peroxidase, an enzyme that protects against oxidative tissue damage. It has a suppressive action on cell proliferation at high levels.

\section{Other bioactive compounds in whole grains}

\section{Lignans}

Lignans are hormonally-active compounds in grains that may protect against hormonally-mediated diseases (Adlercreutz \& Mazur, 1997). Lignans are compounds possessing a 2,3dibenzylbutane structure that are present as minor constituents of many plants, where they form the building blocks for 
the formation of lignin in the plant cell wall. The plant lignans secoisolariciresinol and matairesinol are converted by human gut bacteria to the mammalian lignans enterolactone and enterodiol. Limited information exists on the concentration of lignans and their precursors in food. Due to the association of lignan excretion with fibre intake, it is assumed that plant lignans are contained in the outer layers of the grain. Concentrated sources of lignans include wholegrain wheat, wholegrain oats and rye meal. Seeds that are also concentrated sources of lignans include flaxseed seeds (the most concentrated source), pumpkin seeds, caraway seeds and sunflower seeds. These compositional data suggest that wholegrain breads and cereals are the best means of delivering lignans in the diet.

Grains and other high-fibre foods increase urinary lignan excretion, which is an indirect measure of lignan content in foods (Borriello et al. 1985). Mammalian lignan production of plant foods was studied by Thompson et al. (1996), who used an in vitro fermentation method with human faecal microbiota. Oilseeds, particularly flaxseed flour and meal, produced the highest concentration of lignans, followed by dried seaweeds, whole legumes, cereal brans, wholegrain cereals, vegetables and fruits. Lignan production from flaxseed was approximately 100 times greater than that produced from most other foods.

Differences in the metabolism of phyto-oestrogens among individuals have been noted. Adlercreutz et al. (1986) found total urinary lignan excretion in Finnish women to be positively correlated with total fibre intake, total fibre intake per $\mathrm{kg}$ body weight and grain fibre intake per $\mathrm{kg}$ body weight. Similarly, the geometric mean excretion of enterolactone was positively correlated with the geometric mean intake of dietary grain products $(\mathrm{kJ} / \mathrm{d})$ for five groups of women ( $r$ 0.996).

The association between lignan excretion and fibre intake suggests that plant lignans are probably concentrated in the outer layers of the grain. As current processing techniques eliminate this fraction of the grain, lignans may not be found in processed grain products on the market and would only be found in wholegrain foods.

Serum enterolactone was measured in a cross-sectional study in Finnish adults (Kilkkinen et al. 2001). In men serum enterolactone concentrations were positively associated with consumption of wholegrain products. Variability in serum enterolactone concentration was great, suggesting the role of gut microflora in the metabolism of lignans may be important.

\section{Phytosterols}

Plant sterols and stanols are found in oilseeds, grains, nuts and legumes. These compounds are known to reduce serum cholesterol (Yankah \& Jones, 2001). Structurally, they are very similar to cholesterol, differing in side-chain methyl and ethyl groups. It is believed that phytosterols inhibit dietary and biliary cholesterol absorption from the small intestine. Phytosterols have better solubility than cholesterol in bilesalt micelles in the small intestine. Phytosterols displace cholesterol from micelles, which reduces cholesterol absorption and increases its excretion (Hallikainen et al. 2000). In order to inhibit absorption of dietary cholesterol the sterol must be consumed at the same time as cholesterol. The amount of plant sterols and stanols necessary to achieve a significant cholesterol-lowering effect has been the subject of debate. Although a significant effect has been reported for $<1 \mathrm{~g} / \mathrm{d}$, intakes of $1-2 \mathrm{~g} / \mathrm{d}$ are usually suggested. A doseresponse effect is reported for phytosterols that plateaus at about $2.5 \mathrm{~g} / \mathrm{d}$ (Nair et al. 1984). The average Western diet contains an estimated 200-300 mg plant sterols/d. Vegetarians may consume up to $500 \mathrm{mg} / \mathrm{d}$. Increased whole-grain consumption would increase total phytosterol intake and potentially contribute to cholesterol reduction.

\section{Unsaturated fatty acids}

Wholegrain wheat contains about $30 \mathrm{~g}$ lipids/kg and wholegrain oats contain about $75 \mathrm{~g}$ lipids $/ \mathrm{kg}$. Grain lipids comprise about $75 \mathrm{~g}$ unsaturated fatty acids $/ 100 \mathrm{~g}$, of which there are approximately equal amounts of oleic and linoleic acid and 1-2 g linolenic acid/100 g. Palmitate is the main saturated fatty acid. There are approximately $20 \mathrm{~g}$ unsaturated lipid/ $\mathrm{kg}$ whole wheat and about $55 \mathrm{~g} / \mathrm{kg}$ whole-oat foods. Both oleic and linoleic acid are known to reduce serum cholesterol and are important components of a heart healthy diet (McPherson \& Spiller, 1995). There has been considerable emphasis on low-fat diets for reduced heart disease. However, the type of fat consumed is important as well as the amount of fat. If the fat is saturated, LDL-cholesterol and total cholesterol levels increase, but these levels decrease when the fat is unsaturated. In studies with individual fatty acids stearic acid, oleic acid and linoleic acid were associated with lowering total cholesterol and LDL-cholesterol. Other studies have confirmed the cholesterol-lowering effect of grain lipids and high-lipid bran products (Gerhardt \& Gallo, 1998).

\section{Anti-nutrients}

Anti-nutrients found in grains include digestive enzyme (protease and amylase) inhibitors, phytic acid, haemagglutinins and phenolics and tannins. Protease inhibitors, phytic acid, phenolics and saponins have been shown to reduce the risk of cancer of the colon and breast in animals. Phytic acid, lectins, phenolics, amylase inhibitors and saponins have also been shown to lower the plasma glucose, insulin and/or plasma cholesterol and triacylglycerols (Slavin et al. 1999). In grains protease inhibitors make up 5-10\% of the water-soluble protein and are concentrated in the endosperm and embryo.

\section{Conclusion}

Whole grains are rich in many components, including dietary fibre, starch, fat, antioxidant nutrients, minerals, vitamins, lignans and phenolic compounds that have been linked to reduced risk of $\mathrm{CHD}$, cancer, diabetes, obesity and other chronic diseases. Most of the components are found in the germ and bran, which are reduced in the grain-refining process. The most potent protective components of whole grains need identification so that efforts can be directed to minimising the losses of physiologically-important constituents of grains during processing. There is also a need to educate the public to increase their intake of whole grains to the recommended levels. 


\section{References}

Adlercreutz H, Fotsis T, Bannwart C, Hamalainen E, Bloigu A \& Ollus A (1986) Urinary estrogen profile determination in young Finnish vegetarian and omnivorous women. Journal of Steroid Biochemistry 24, 289-296.

Adlercreutz H \& Mazur W (1997) Phyto-oestrogens and western diseases. Annals of Medicine 29, 95-120.

Bjorck I, Granfeldt Y, Lilijeberg H, Tovar J \& Asp N (1994) Food properties affecting the digestion and absorption of carbohydrates. American Journal of Clinical Nutrition 59S, 688S-705S.

Borriello SP, Setchell KD, Axelson M \& Lawson AM (1985) Production and metabolism of lignans by the human faecal flora. Journal of Applied Bacteriology 58, 37-43.

Gerhardt AL \& Gallo NB (1998) Full-fat rice bran and oat bran similarly reduce hypercholesterolemia in humans. Journal of Nutrition 128, 865-869.

Gibson GR, Beatty ER, Wang X \& Cummings JH (1995) Selective stimulation of bifidobacteria in the human colon by oligofructose and inulin. Gastroenterology 108, 975-982.

Giovannucci E (1995) Insulin and colon cancer. Cancer Causes \& and Control 6, 164-179.

Granfeldt Y, Hagander B \& Bjorck I (1995) Metabolic responses to starch in oat and wheat products. On the importance of food structure, incomplete gelatinization or presence of viscous dietary fibre. European Journal of Clinical Nutrition 49, 189-199.

Hallikainen MA, Sarkkinen ES \& Uusitupa MIJ (2000) Plant stanols esters affect serum cholesterol concentrations of hypercholesterolemic men and women in a dose-dependent manner. Journal of Nutrition 130, 767-776.

Heaton KW, Marcus SN, Emmett PM \& Bolton CH (1988) Particle size of wheat, maize, and oat test meals: effects on plasma glucose and insulin responses and on the rate of starch digestion in vitro. American Journal of Clinical Nutrition 47, 675-682.

Hu FB, Manson JE, Liu S, Hunter D, Colditz GA, Michels KB, Speizer FE \& Giovannucci E (1999) Prospective study of adult onset diabetes mellitus (type 2) and risk of colorectal cancer in women. Journal of the National Cancer Inst Institute 91, 542-547.

Jang Y, Lee JH, Kim OY, Park HY \& Lee SY (2001) Consumption of whole grain and legume powder reduces insulin demand, lipid peroxidation, and plasma homocysteine concentrations in patients with coronary artery disease: randomized controlled clinical trial. Arteriosclerosis Thrombosis and Vascular Biology 21, 2065-2071.

Jarvi A, Karlstrom B, Granfeldt YE, Bjorck I \& Vessby B (1995) The influence of food structure on postprandial metabolism in patients with non-insulin-dependent diabetes mellitus. American Journal of Clinical Nutrition 61, 837-842.

Jenkins DJ, Wesson V, Wolever TM, Jenkins AL, Kalmusky J, Guidici S, Csima A, Josse RG \& Wong GS (1988) Wholemeal versus wholegrain breads: proportion of whole or cracked grain and the glycaemic response. British Medical Journal 297, 958-960.

Jenkins DJA, Wolever TMS, Jenkins AL, Giordano C, Giudici S, Thompson LU, Kalmusky J, Josse RG \& Wong GS (1986) Low glycemic response to traditionally processed wheat and rye products: bulgur and pumpernickel bread. American Journal of Clinical Nutrition 43, 516-520.

Juntunen KS, Niskanen LK, Liukkonen KH, Poutanen KS, Holst JJ \& Hykkanen HM (2002) Postprandial glucose, insulin, and incretin responses to grain products in healthy subjects. American Journal of Clinical Nutrition 75, 254-262.

Katz DL, Nawaz H, Boukhalil J, Chan W, Ahmadi R, Giannamore V \& Sarrel PM (2001) Effects of oat and wheat cereals on endothelial responses. Preventative Medicine 33, 476-484.

Kilkkinen A, Stumpf K, Pietinen P, Valsta LM, Tapanainen H \& Adlercreutz H (2001) Determinants of serum enterolactone concentration. American Journal of Clinical Nutrition 73, 1094-1100.

Kroon PA, Faulds CB, Ryden P, Robertson JA \& Williamson G (1997) Release of covalently bound ferulic acid from fiber in the human colon. Journal of Agricultural and Food Chemistry 45, 661-667.

McIntyre A, Vincent RM, Perkins AC \& Spiller RC (1997) Effect of bran, ispaghula, and inert plastic particles on gastric emptying and small bowel transit in humans: the role of physical factors. Gut 40, 223-227.

McPherson R \& Spiller GA (1995) Effects of dietary fatty acids and cholesterol on cardiovascular disease risk factors in man. In Handbook of Lipids in Human Nutrition, pp. 41-49 [GA Spiller, editor]. Boca Raton, FL: CRC Press.

Marlett JA, McBurney MI \& Slavin JL (2002) Position of the American Dietetic Association: Health implications of dietary fiber. Journal of the American Dietetic Association 102, 993-1000.

Miller G (2001) Whole grain, fiber and antioxidants. In CRC Handbook of Dietary Fiber in Human Nutrition, pp. 453-460 [GA Spiller, editor]. Boca Raton, FL: CRC Press.

Nair PP, Turjmann N, Kessic G, Calkins B, Goodman GT, Davidovitz H \& Nimmagadda G (1984) Diet, nutrition intake and metabolism in populations at high and low risk for colon cancer. American Journal of Clinical Nutrition 40, 927-930.

Pereira MA, Jacobs DJ, Pins JJ, Raatz SK, Gross MD, Slavin JL \& Seaquist ER (2002) Effect of whole grains on insulin sensitivity in overweight hyperinsulinemic adults. American Journal of Clinical Nutrition 75, 848-855.

Pereira MA, Pins JJ, Jacobs DR, Marquart L \& Keenan JM (2001) Whole grains, cereal fiber and chronic diseases: Epidemiologic evidence. In CRC Handbook of Dietary Fiber in Human Nutrition, pp. 461-479 [GA Spiller, editor]. Boca Raton, FL: CRC Press.

Pins JJ, Geleva D, Leemam K, Fraze C, O'Connor PJ \& Cherney LM (2002) Do whole-grain oat cereals reduce the need for antihypertensive medications and improve blood pressure control? Journal of Family Practice 51, 353-359.

Schoen RE, Tangen CM, Kuller LH, Burke GL, Cushman M, Tracy RP, Dobs A \& Savage PJ (1999) Increased blood glucose and insulin, body size and incident colorectal cancer. Journal of the National Cancer Institute 91, 1147-1154.

Slavin JL, Jacobs D \& Marquart L (2001a) Grain processing and nutrition. Critical Reviews in Biotechnology 21, 49-66.

Slavin JL, Jacobs D, Marquart L \& Wiemer K (2001b) The role of whole grains in disease prevention. Journal of the American Dietetic Association 101, 780-785.

Slavin JL, Martini MC, Jacobs DR \& Marquart L (1999) Plausible mechanisms for the protectiveness of whole grains. American Journal of Clinical Nutrition 70, 459S-463S.

Thompson LU, Seidl MM, Rickard SE, Orcheson LJ \& Fong HH (1996) Antitumorigenic effect of a mammalian lignan precursor from flaxseed. Nutrition and Cancer 26, 159-165.

Truswell AS (2002) Cereal grains and coronary heart disease. European Journal of Clinical Nutrition 56, 1-14.

Van Dam RM, Rimm EB, Willett WC, Stampfer MJ \& Hu FB (2002) Dietary patterns and risk for type 2 diabetes mellitus in US men. Annals of Internal Medicine 136, 201-209.

Van Loo J, Coussement P, De Leenheer L, Hoebregs H \& Smits G (1995) On the presence of inulin and oligofructose as natural ingredients in the western diet. Critical Reviews in Food Science and Nutrition 35, 525-552.

Wrick K, Robertson JB \& Van Soest PJ (1983) The influence of dietary fiber source on human intestinal transit and stool output. Journal of Nutrition 113, 1464-1479.

Yankah VV \& Jones PJH (2001) Phytosterols and health implications - efficacy and nutritional aspects. Inform 12, 899-903. 